\title{
Pharmacological treatment for unipolar psychotic
} depression

\author{
Systematic review and meta-analysis
}

\author{
JAAP WIJKSTRA, JEROEN LIJMER, FERDI J. BALK, JOHN R. GEDDES \\ and WILLEM A. NOLEN
}

\author{
Background The optimal \\ pharmacological treatment of unipolar \\ psychotic depression is uncertain.
}

\begin{abstract}
Aims To compare the clinical effectiveness of pharmacological treatments for patients with unipolar psychotic depression.
\end{abstract}

Method Systematic review and metaanalysis of randomised controlled trials.

\begin{abstract}
Results Ten trials were included in the review. We found no evidence that the combination of an antidepressant with an antipsychotic is more effective than an antidepressant alone. This combination was statistically more effective than an antipsychotic alone.
\end{abstract}

\section{Conclusions Antidepressant mono-} therapy and adding an antipsychotic if the patient does not respond, or starting with the combination of an antidepressant and an antipsychotic, both appear to be appropriate options for patients with unipolar psychotic depression. However, clinically the balance between risks and benefits may suggest the first option should be preferred for many patients. Starting with an antipsychotic alone appears to be inadequate.

\section{Declaration of interest J.W. and}

W.N. are currently conducting a randomised controlled trial in patients with unipolar psychotic depression, which is financially supported by Wyeth and AstraZeneca. These organisations also financially supported the literature search for this review.
For unipolar psychotic depression, in which psychotic features appear in the context of a major depressive episode, electroconvulsive therapy (ECT) is considered by many clinicians to be the most effective and therefore the first-line treatment. Pharmacotherapy is also often considered a suitable first-line treatment, because many patients prefer drug therapy to ECT and moreover, after a successful course of ECT subsequent treatment with medication is often needed to prevent relapse. If the choice is pharmacotherapy, it is unclear whether one can start with an antidepressant alone or should combine it with an antipsychotic. Some reviews suggest that one may consider antidepressant monotherapy before adding an antipsychotic (Nelson \& McElroy, 1997; Wheeler-Vega et al, 2000). However, recent US and British guidelines recommend the combination (American Psychiatric Association, 2000; National Institute for Clinical Excellence, 2004). We report a systematic review of the evidence regarding the pharmacological treatment of unipolar psychotic depression.

\section{METHOD}

This review was performed as a Cochrane systematic review in cooperation with the Cochrane Collaboration Depression, Anxiety and Neurosis Group, London (Wijkstra et al, 2005).

\section{Included studies}

We included randomised controlled trials (RCTs) of the pharmacological treatment of patients with psychotic depression, published in any language. We expected to identify very few RCTs with the treatment of psychotic depression as the primary focus. We therefore also selected RCTs including patients with major depression with and without psychotic features, in which the effects in the subgroup of patients with psychotic depression were reported separately. The inclusion criteria for the review were as follows.

\section{Participants}

We included RCTs investigating patients in any setting (in-patient and out-patient) with a unipolar major depressive disorder having a current major depressive episode with psychotic features. If a trial had studied patients with depressive episodes in the course of a bipolar disorder, it was only included if the results in the non-bipolar depression group were reported separately or if the percentage of patients with bipolar depression did not exceed $20 \%$ of the total study population.

\section{Interventions}

We included RCTs making the following comparisons: antidepressant $v$. antidepressant, antipsychotic $v$. antipsychotic, antidepressant $v$. placebo, antipsychotic $v$. placebo, antidepressant $v$. antipsychotic, antidepressant plus antipsychotic $v$. antidepressant, antidepressant plus antipsychotic $v$. antipsychotic, antidepressant plus antipsychotic $v$. placebo.

\section{Search strategy for identification of studies}

Bibliographic databases such as Medline do not have an indexing term for psychotic depression. We therefore screened all RCTs that had included patients with a unipolar major depressive disorder to identify those possibly including patients with psychotic features. We searched the Cochrane Central Register of Controlled Trials with the terms DEPRESSIVE DISORDER and DRUG TREATMENT. In addition we searched Medline (1966 until April 2004) and EMBASE (1980 until April 2004) using the following terms: ('DEPRESSIVE DISORDER/DRUG THERAPY' [MESH] and (('DELUSIONS' [MESH Terms] or DELUSIONS [Text Word]) or (('PSYCHOTIC DISORDERS' [MESH Terms] or PSYCHOTIC [Text Word]) and FEATURES [All Fields]))) combined with a search strategy for RCTs.

In step 1 of the search process, all abstracts of the identified publications were screened independently by two authors (50\% by both J.W. and J.L., $50 \%$ by both F.B. and W.N.) and studies were selected if they met the following criteria:

(a) the study was a randomised controlled trial; 
(b) included patients with a major depressive disorder;

(c) investigated the effectiveness of pharmacological treatment; and

(d) concerned acute-phase treatment.

In case of any doubt or disagreement between the reviewers, the publication was included. Next, the full articles were obtained for the selected abstracts. In step 2, a trained medical student screened the full articles to select all trials in which: (a) patients with psychotic depression were not excluded; and (b) results in the subgroup of patients with psychotic depression were reported separately.

In case of any doubt the publication was included. In order to check the reliability of this procedure a random selection of 60 articles were also screened by J.W., which revealed no publication that had not been selected by the medical student. In addition, reference lists of included publications, related reviews and abstract books of recent congresses were searched and trials were identified through personal communication. In step 3, two authors (J.W. and F.B.) independently reviewed all identified publications according to the inclusion criteria. Any disagreement was resolved by consensus discussion with a third author (W.N.).

\section{Quality assessment}

Two reviewers (J.W. and J.L.) assessed the methodological quality of the included trials, according to the criteria of the Cochrane Collaboration. These criteria focus on randomisation procedures (especially allocation concealment and randomisation); whether the study was double-blind, single-blind or open randomised; analysis (stratification prior to treatment or non-stratification of patients with psychotic $v$. non-psychotic depression in the RCTs that did not have the treatment of psychotic depression as their main focus); and other aspects, such as reporting of the number of patients leaving the trial and the reasons for the withdrawals.

\section{Types of outcome measures}

The primary efficacy outcome used in the analysis was clinical response based on observer-rated symptom reduction, for example a reduction of at least $50 \%$ on the Hamilton Rating Scale for Depression (HRSD) or any other observer-rated depression severity rating scale, or a change score on the Clinical Global Impression -
Change (CGI-C) of 'much improved' or 'very much improved'. As secondary efficacy outcomes, we investigated remission as defined in the reports and based on the HRSD or other observer-rated depression severity scale or change in severity on Clinical Global Impression - Severity (CGI-S); and quality of life.

The primary harm outcome used in the analysis was overall withdrawal rate during acute treatment as a proxy measure of overall acceptability of treatment. We also analysed withdrawal rates resulting from adverse effects, all-cause mortality and suicide.

\section{Data extraction}

Data were extracted on participants' characteristics, diagnosis (diagnostic instrument, classification), intervention details and outcome measures. Data were extracted independently by two reviewers (J.W. and J.L.).

\section{Data analysis}

Data were entered into RevMan 4.2 (http:// www.cc-ims.net/RevMan). For binary efficacy outcomes a relative risk (with $95 \%$ confidence intervals) was calculated for each comparison. When necessary, we converted response data from the trials into intention-to-treat response data by using the total number of randomised patients per group who had started with treatment as the denominator.

\section{RESULTS}

\section{Description of the studies}

From the search in the Cochrane Central Register of Controlled Trials we identified 1782 publications. The searches in Medline and EMBASE resulted in 720 and 831 publications respectively. The first step of screening the abstracts of these publications resulted in 789 publications (749 from the Cochrane register, 38 from Medline and 11 from EMBASE). The second step of screening the full articles resulted in the identification of 52 publications $(47,3$ and 2 respectively). Hand-searching of reference lists of relevant reviews resulted in one further publication (Bellini et al, 1994), whereas hand-searching of the included publications revealed no other publication. The third step of reviewing these 53 publications resulted in seven included studies. Finally, we added two other publications which we knew were then in press: one by Van den Broek et al
(2004) and one by Rothschild et al (2004), reporting two similar trials. Thus, nine publications with a total of ten RCTs were included (Table 1).

In seven of the ten studies the treatment of psychotic depression was the primary focus. From three studies we used data from the subgroup of patients with psychosis, which were reported separately (Spiker \& Kupfer, 1988; Bruijn et al, 1996; Van den Broek et al, 2004). Five RCTs did not include only patients with unipolar psychotic depression. In the study by Zanardi et al (1996) it was possible to exclude the data relating to participants with bipolar disorder. The study by Anton \& Burch (1990) reported $15.8 \%$ (6 out of 38 ) cases of bipolar disorder, and it is unclear how many of the 8 participants who left the study and whose data were excluded before analysis had bipolar disorder. To solve this problem we assumed a random withdrawal rate. In Spiker et al (1985) 15.5\% of the patients in the results had bipolar disorder. In Bruijn et al (1996) and Zanardi et al (2000) we were able to exclude the data for patients with bipolar disorder with the help of additional information from the authors.

\section{Outcome measures}

It was not possible to transfer the authors' defined response data into rates based on one definition (e.g. $50 \%$ reduction of the HRSD score). In addition, several authors used response definitions based on what is often considered remission. In the absence of a better option, we decided to use only response data as reported by the authors.

In eight of the ten studies we recalculated the intention-to-treat response rates using all randomised patients as the denominator. We thus included many patients who were excluded from analyses by the original researchers: from the study of Anton \& Burch (1990), 8 patients who left the study before receiving 2 full weeks of active medication; 9 and 3 patients, respectively from the studies of Bruijn et al (1996) and Van den Brock et al (2004), who were treated with haloperidol; from the study of Mulsant et al (2001), 6 patients who left the trial after randomisation; $7 \%$ and $9 \%$ of the randomised patients respectively from the two trials of Rothschild et al (2004), who left the trial between baseline and the first visit after start of treatment at week 1; and finally 7 patients who left the studies of Spiker et al (1985) and Spiker \& Kupfer (1988). Extracting continuous 


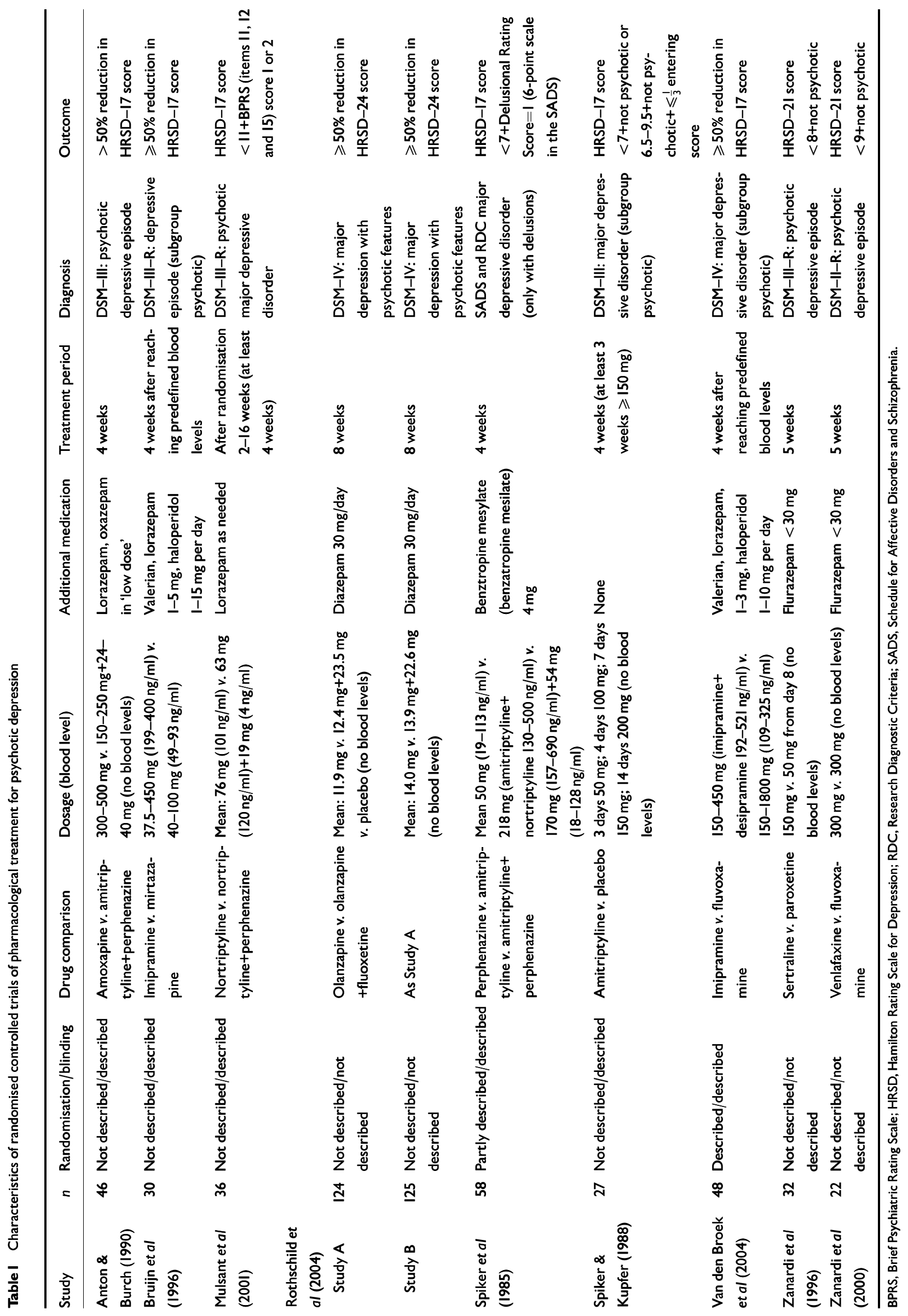


data of observer-rated depression severity scales for analysis was impossible because we were not able to convert these data according to an intention-to-treat analysis (Spiker et al, 1985; Spiker \& Kupfer, 1988; Anton \& Burch, 1990; Bruijn et al, 1996; Mulsant et al, 2001; Rothschild et al, 2004; Van den Broek et al, 2004), and in the two studies by Zanardi et al (1996, 2000) no continuous data were given. Other efficacy outcome measures (e.g. change in quality of life) could not be extracted from the trials.

Overall rates of withdrawal were available for all studies. Rates of withdrawal because of adverse effects were available in four studies (Spiker et al, 1985; Anton \& Burch, 1990; Mulsant et al, 2001; Van den Broek et al, 2004); in three other studies these data were not based on an intention-totreat analysis (two in Rothschild et al, 2004; one in Bruijn et al, 1996), were not available in one study (Spiker \& Kupfer, 1988) and were the same as the overall withdrawal rates in two studies (Zanardi et al, 1996, 2000). Withdrawals specifically owing to death or suicide were not reported in any of the studies.

\section{Efficacy analyses}

Only one RCT compared an antidepressant with a placebo (Spiker \& Kupfer, 1988). In this study amitriptyline was not statistically significantly more effective than placebo $(\mathrm{RR}=8.40,95 \%$ CI $0.50-142.27 ; P=0.14)$. In four studies two different antidepressants were compared directly. In one study (Bruijn et al, 1996), imipramine under plasma level control was statistically significantly more effective than mirtazapine $\quad(\mathrm{RR}=3.00, \quad 95 \% \quad \mathrm{CI} \quad 1.01-8.95$; $P=0.05$ ). In another (Van den Broek et al, 2004), imipramine under plasma level control was statistically significantly more effective than fluvoxamine $(\mathrm{RR}=2.10$, $95 \%$ CI 1.06-4.17; $P=0.03)$. In the first study by Zanardi et al (1996), sertraline was statistically significantly more effective than paroxetine $(\mathrm{RR}=3.37,95 \% \quad \mathrm{CI}$ 1.19-9.57; $P=0.02$ ); the second (Zanardi et al, 2000) did not find a statistically significant difference between fluvoxamine and venlafaxine.

In two studies the tricyclic antidepressant (TCA) imipramine given under plasma level control was compared with an antidepressant of another class (mirtazapine or fluvoxamine). After pooling these studies (Bruijn et al, 1996; Van den Broek et al, 2004)

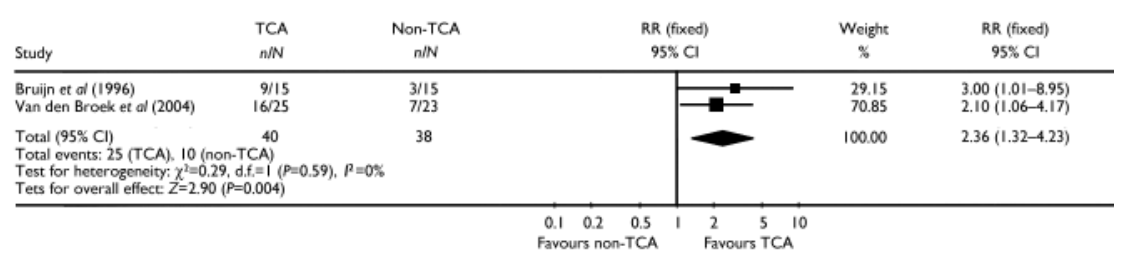

Fig. I Efficacy of tricyclic antidepressant (TCA) monotherapy v. non-tricyclic antidepressant (non-TCA) monotherapy (study-defined outcome).

imipramine was statistically significantly superior to the non-TCA (RR=2.36, 95\% CI 1.32-4.23, $P=0.004$ ) (Fig. 1).

In three RCTs selective serotonin reuptake inhibitors (SSRIs) were studied. Response rates to these SSRIs varied from 21.4\% (paroxetine in Zanardi et al, 1996) and $30.4 \%$ (fluvoxamine in Van den Broek et al, 2004) to $72.2 \%$ (sertraline in Zanardi et al, 1996) and $81.8 \%$ (fluvoxamine in Zanardi et al, 2000). In one of these studies (Zanardi et al, 1996) there was a statistically significant difference between two SSRIs, favouring sertraline. Combining the studies with SSRIs led to a mean response rate to SSRIs of $51.5 \%$. A pooled comparison of SSRIs with other antidepressants was not possible.

One study (Spiker et al, 1985) comparing antidepressant monotherapy (amitriptyline) with antipsychotic monotherapy (perphenazine) did not find a statistically significant difference $(\mathrm{RR}=2.09,95 \% \mathrm{CI}$ 0.64-6.82; $P=0.22$ ).

We found two studies comparing antipsychotic monotherapy (olanzapine) with placebo (Rothschild et al, 2004). Pooling these studies did not show a statistically significant difference $(\mathrm{RR}=1.13,95 \% \mathrm{CI}$ 0.74-1.73; $P=0.57$ ).

In two studies the combination of an antidepressant (nortriptyline or amitriptyline) and an antipsychotic (perphenazine) was compared with antidepressant monotherapy (Spiker et al, 1985; Mulsant et al, 2001). Pooling these two studies did not show a statistically significant difference between a TCA plus an antipsychotic and a TCA alone $(\mathrm{RR}=1.44,95 \%$ CI $0.86-$ $2.41 ; P=0.16$ ) (Fig. 2).

In three studies the combination of an antidepressant and an antipsychotic was compared with antipsychotic monotherapy. In one of these studies (Spiker et al, 1985) the combination of amitriptyline plus perphenazine was statistically significantly superior to perphenazine alone $(\mathrm{RR}=3.61$, $95 \%$ CI $1.23-10.56 ; P=0.02$ ). In the other two studies comparing the combination of olanzapine plus fluoxetine (Rothschild et al, 2004) with olanzapine alone, pooling resulted in a significant advantage for the combination over the antipsychotic alone $(\mathrm{RR}=1.64,95 \%$ CI $1.10-2.44 ; P=0.01)$ and over placebo $(\mathrm{RR}=1.86,95 \% \mathrm{CI}$ 1.23-2.82; $P=0.003$ ). Pooling the data from all three studies comparing the combination of an antidepressant plus an antipsychotic with an antipsychotic alone showed a statistically significant difference favouring the combination $(\mathrm{RR}=1.92$, 95\% CI 1.32-2.80; $P=0.0007$ ) (Fig. 3).

\section{Other analyses}

The rates of withdrawal from the studies varied from $9 \%$ to $41 \%$. In the two multicentre trials with olanzapine/fluoxetine (Rothschild et al, 2004) the rate was 102 out of $249(41 \%)$, and these authors reported even higher non-completion rates (completers: 110 out of $249=44 \%$, thus the non-completion rate was $56 \%$ ). There was no statistically significant difference in the overall withdrawal rates between any of the treatments, either in the individual studies or after pooling of studies.

\section{DISCUSSION}

Despite our extensive search of the literature, we identified very few RCTs investigating the pharmacological treatment of patients with a unipolar major depressive episode with psychotic features (psychotic depression). In addition to seven trials in which the treatment of patients with psychotic depression was a major focus of the study, we were able to find three other trials that reported on the effects in a subgroup of patients with psychotic depression separately. The authors of two of these studies of both psychotic and non-psychotic depression provided us with additional information on the results in the subgroups of patients with psychotic depression. Because of the numbers involved, we were not able to approach the authors of all RCTs comprising depressed patients to request similar information. However, if 


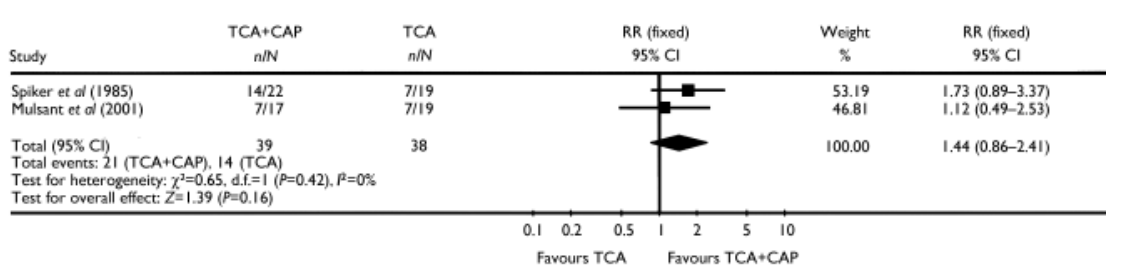

Fig. 2 Efficacy of the combination of a tricyclic antidepressant (TCA) plus a classical antipsychotic (CAP) $v$. TCA monotherapy (study-defined outcome).

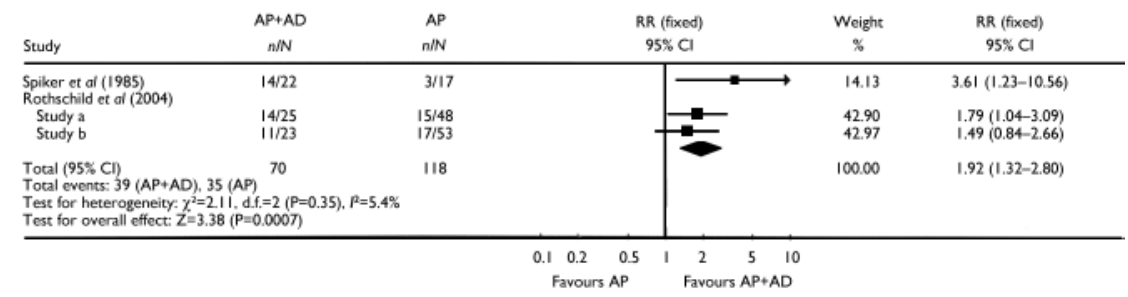

Fig. 3 Efficacy of the combination of an antipsychotic (AP) plus an antidepressant (AD) v. antipsychotic monotherapy (study-defined outcome).

data from other RCTs on the subgroup of people with psychotic depression are available, we invite the authors of these trials to provide us with the relevant data, so that we may update this systematic review.

\section{Underinvestigation of unipolar psychotic depression}

That we identified only ten RCTs in psychotic depression illustrates that this most severe form of depression is seriously underinvestigated. One probable reason for this is that it is difficult to conduct RCTs in patients with psychotic depression. These patients not only have a psychotic illness, but often also are very anxious or physically ill. In addition, they are often offered ECT directly because many clinicians assume that ECT is more effective than pharmacotherapy. Patients with psychotic depressive illness may be unable to give informed consent or may tend to withdraw from trials. Furthermore - until the recent trials by Rothschild et al (2004) pharmaceutical companies were not interested in conducting trials in psychotic depression because this subgroup of depression is not considered a separate indication for treatment by the regulatory authorities and therefore is commercially unattractive.

\section{Implications of the study}

Despite the paucity of RCTs, a few clinically relevant conclusions can be drawn. First, there is no evidence for the clinical belief that an antidepressant alone is ineffective in psychotic depression. In seven of the ten studies there was at least one treatment arm with an antidepressant as monotherapy, with in total 11 treatment arms. In 5 of these treatment arms the antidepressant was effective in more than $50 \%$ of the patients: imipramine in Bruijn et al (1996) and Van den Broek et al (2004), sertraline in Zanardi et al (1996) and fluvoxamine and venlafaxine in Zanardi et al (2000). In three studies there was even a statistically significant difference between two antidepressants. In two of these studies imipramine (under plasma level control) was more effective than fluvoxamine (Van den Broek et al, 2004) and mirtazapine (Bruijn et al, 1996) respectively, suggesting that a tricyclic antidepressant is to be preferred over a non-tricyclic drug in patients with psychotic depression. This finding is in line with three studies among hospitalised, depressed patients in which clomipramine was found to be more effective than citalopram, paroxetine or moclobemide respectively (Danish University Antidepressant Group, 1986, 1990, 1993). In these three studies patients with psychotic depression were also included; unfortunately, however, it is not possible to identify which patients these were, as this information was not systematically recorded. In the third trial finding a difference between two antidepressants (Zanardi et al, 1996), more patients responded to sertraline than to paroxetine, probably related to more patients withdrawing from the paroxetine group. It is difficult to draw a conclusion from this study, as in another study (Zanardi et al, 2000) the same group found good response rates to another SSRI, fluvoxamine, as well as to venlafaxine.

Second, there is no evidence that the combination of an antidepressant with an antipsychotic is more effective than an antidepressant alone. Therefore, it can be concluded that the recommendation in the US and British guidelines (American Psychiatric Association, 2000; National Institute for Clinical Excellence, 2004) that in psychotic depression the combination therapy should be preferred over an antidepressant alone is not reliably evidence-based, if not necessarily incorrect. Clinically, the balance between risks and benefits may suggest that initial monotherapy with an antidepressant should be the preferred option for many patients.

Finally, there is evidence that the combination of an antidepressant with an antipsychotic is more effective than an antipsychotic alone. This was the major result of the study comparing amitriptyline plus perphenazine $v$. perphenazine alone (Spiker et al, 1985) and was also found in one of the studies comparing fluoxetine plus olanzapine $v$. olanzapine alone (Rothschild et al, 2004). Moreover, it was confirmed in the pooled analysis of these studies. Therefore, it is concluded that treatment should not begin with antipsychotic monotherapy.

\section{Limitations of the study}

Our review has several limitations. First, none of the studies with antidepressant monotherapy had a sample size exceeding 25 patients per group. The only two relative large studies were the studies sponsored by Eli Lilly (Rothschild et al, 2004) with around 50 patients per group (olanzapine 48 and 53 patients, and placebo 51 and 49 patients respectively), but with fewer patients in the group receiving olanzapine plus fluoxetine ( 25 and 23 respectively). As with all systematic reviews, publication bias is a potentially serious source of error. There were too few studies - especially too few larger studies to investigate further the possibility of publication bias, and so it cannot be ruled out. Additionally, the relative high proportion of these small studies (5 out of 10) reporting a significant difference between two treatments suggests publication bias.

Second, we could only use one outcome measure regarding efficacy: the response rates as defined by the authors. It was impossible to recalculate these response rates into a standard rate based on one definition (e.g. HRSD score), as many studies used different versions of the HRSD or actually reported only remission rates. As some of these authors' response definitions may 
actually be considered remission, this might have had an influence on the results of our meta-analysis.

Finally, there was considerable clinical heterogeneity between the trials, illustrated by substantial differences in response rates to antidepressant monotherapy between the European and the US studies. Two Italian studies (Zanardi et al, 1996, 2000) reported high response rates (above $50 \%$ ) to SSRIs (with the exception of paroxetine), and in the Dutch studies (Bruijn et al, 1996; Van den Broek et al, 2004) the response rate was above $50 \%$ to imipramine (but not to mirtazapine and fluvoxamine). In contrast, the US studies reported response rates below $50 \%$ (Spiker et al, 1985; Spiker \& Kupfer, 1988; Mulsant et al, 2001). One likely reason for this USEuropean discrepancy is differences between the study populations. Although all studies required that patients fulfilled diagnostic criteria according to a specified diagnostic classification, the reliability of diagnosis may have been limited in some - if not most - of the trials. Only four trials used a semi-structured interview (Spiker et al, 1985; Bruijn et al, 1996; Mulsant et al, 2001; Van den Broek et al, 2004), and only one of these trials (Bruijn et al, 1996) reported the specific psychotic features for all patients. This leaves open the possibility that the conclusion that in a particular patient (for instance) a feeling of guilt was actually a delusion was drawn differently across the trials in this review. A similar problem may have played a part in the judgement as to whether a patient had a psychotic depression in the course of unipolar disorder or bipolar disorder.

\section{ACKNOWLEDGEMENTS}

The authors are grateful for the support from the Cochrane Collaboration Depression, Anxiety and Neurosis Group. We thank Dr J. Bruijn, Dr W. Van den Broek and Dr R. Zanardi for additional data from their studies.

\section{REFERENCES}

Anton, R. F. \& Burch, E. A. (1990) Amoxapine versus amitriptyline combined with perphenazine in the treatment of psychotic depression. American Journal of Psychiatry, 147, 1203-1208.

American Psychiatric Association (2000) Practice Guideline for the Treatment of Patients with Major Depressive Disorder (Revision). Washington, DC: APA.

Bellini, L., Gasperini, M., Gatti, F., et al (1994) A double blind study with fluvoxamine vs. desipramine combined with placebo or haloperidol in delusional depression. Critical Issues in the Treatment of Affective Disorders, 9, 32-36.

\section{CLINICAL IMPLICATIONS}

- Initial antidepressant monotherapy and adding an antipsychotic if the patient does not respond, is an appropriate treatment option for patients with unipolar psychotic depression.

- A tricyclic antidepressant (e.g. imipramine under plasma level control) is probably the best choice in antidepressant monotherapy.

- Starting with antipsychotic monotherapy is not an appropriate treatment strategy.

\section{LIMITATIONS}

- Our conclusions are based on only a few, small randomised controlled trials, and publication bias cannot be ruled out.

- We could only use the outcome measures defined by the authors.

- There was considerable clinical heterogeneity between the trials.

JAAP WIJKSTRA, MD, Rudolf Magnus Institute of Neuroscience, Department of Psychiatry, University Medical Centre, Utrecht, The Netherlands; JEROEN LIJMER, PhD, Department of Psychiatry, Waterland Hospital, Purmerend, The Netherlands; FERDI J. BALK, MD, Rudolf Magnus Institute of Neuroscience, Department of Psychiatry, University Medical Centre, Utrecht, The Netherlands; JOHN R. GEDDES, MD, FRCPsych, Department of Psychiatry, University of Oxford, Warneford Hospital, Oxford, UK; WILLEM A. NOLEN, PhD, University Medical Centre Groningen, Department of Psychiatry, Groningen, The Netherlands

Correspondence: Dr J.Wijkstra, University Medical Centre Utrecht, HP B0I.206, PO Box 85500, 3508 GA, Utrecht, The Netherlands. E-mail: j.wijkstra@azu.nl

(First received 25 February 2005, final revision 23 May 2005, accepted 16 June 2005)

Bruijn, J. A., Moleman, P., Mulder, P. G., et al (1996) A double-blind, fixed blood-level study comparing mirtazapine with imipramine in depressed in-patients. Psychopharmacology, 127, 231-237.

Danish University Antidepressant Group (1986) Citalopram: clinical effect profile in comparison with clomipramine. A controlled multicenter study. Psychopharmacology, 90, 131-138.

Danish University Antidepressant Group (1990) Paroxetine: a selective serotonin reuptake inhibitor showing better tolerance, but weaker antidepressant effect than clomipramine in a controlled multicenter study. Journal of Affective Disorders, 18, 289-299.

Danish University Antidepresasnt Group (1993) Moclobemide: a reversible MAO-A inhibitor showing weaker antidepressant effect than clomipramine in a controlled multicenter study. Journal of Affective Disorders, 28, 105-116.

Mulsant, B. H., Sweet, R. A., Rosen, J., et al (200I) A double-blind randomized comparison of nortriptyline plus perphenazine versus nortriptyline plus placebo in the treatment of psychotic depression in late life. Journal of Clinical Psychiatry, 62, 597-604.

National Institute for Clinical Excellence (2004) Depression: Management of Depression in Primary and Secondary Care. Clinical Guideline 23. London: NICE.

Nelson, E. B. \& McElroy, S. L. (1997) Psychotic depression: a guide to drug choice. CNS drugs, 8, 457-473.

Rothschild, A. J., Williamson, D. J., Tohen, M. F., et al (2004) A double-blind, randomized study of olanzapine and olanzapine/fluoxetine combination for major depression with psychotic features. Journal of Clinical Psychopharmacology, 24, 365-373.

Spiker, D. G.\& Kupfer, D. J. (1988) Placebo response rates in psychotic and nonpsychotic depression. Journal of Affective Disorders, 14, 21-23.

Spiker, D. G., Cofsky, W. J., Dealy, R. S., et al (1985) The pharmacological treatment of delusional depression. American journal of Psychiatry, 42, 430-436.

Van den Broek, W. W., Birkenhäger, T. K., Mulder, P. G. H., et al (2004) A double blind study comparing imipramine with fluvoxamine in depressed inpatients. Psychopharmacology, 175, 48I-486.

Wijkstra, J., Lijmer, J., Balk, F., et al (2005) Pharmacological treatment for psychotic depression. The Cochrane Database of Systematic Reviews, Issue 4. article no. CD004044.pub2. doi: 10.1002/ 1465I858.CD004044.pub2

Wheeler Vega, J. A., Mortimer, A. M., \& Tyson, P. J. (2000) Somatic treatment of psychotic depression: review and recommendations for practice. Journal of Clinical Psychopharmacology, 20, 504-519.

Zanardi, R., Franchini, L., Gasperini, M., et al (1996) Double-blind controlled trial of sertraline versus paroxetine in the treatment of delusional depression. American Journal of Psychiatry, 153, 1631-1633.

Zanardi, R., Franchini, L., Serrett, A., et al (2000) Venlafaxine versus fluvoxamine in the treatment of delusional depression: a pilot double-blind controlled study. Journal of Clinical Psychiatry, 6I, 26-29. 